\title{
Tomografia de coerência óptica na avaliação da camada de fibras nervosas peripapilar nos usuários de cloroquina
}

\author{
Optical coherence tomography to evaluate peripapillary retinal nerve fiber \\ layer in chloroquine patients
}

\author{
Luis Augusto Arana ${ }^{1}$ \\ Jayme Arana ${ }^{2}$ \\ Alexander Rodrigo Hasimoto ${ }^{3}$ \\ Gustavo Schirr ${ }^{4}$ \\ Eduardo Arana ${ }^{5}$ \\ Ana Paula Beckhauser ${ }^{6}$ \\ Francisco Araújo ${ }^{7}$ \\ Thelma Skare ${ }^{8}$
}

Trabalho realizado no Hospital de Olhos do Paraná e no Hospital Evangélico de Curitiba - HUEC - Curitiba (PR) - Brasil.

${ }^{1}$ Fellow de Retina do Doheny Eye Institute - Los Angeles (Califórnia-CA).

${ }^{2}$ Professor Assistente de Oftalmologia do Hospital Evangélico de Curitiba - HUEC - Curitiba (PR) - Brasil.

${ }^{3}$ Residente de Oftalmologia do HUEC - Curitiba (PR) Brasil.

${ }^{4}$ Residente de Oftalmologia do HUEC - Curitiba (PR) Brasil.

${ }^{5}$ Residente de Oftalmologia do HUEC - Curitiba (PR) Brasil.

${ }^{6}$ Residente de Reumatologia do HUEC - Curitiba (PR) Brasil.

${ }^{7}$ Residente de Reumatologia do HUEC - Curitiba (PR) Brasil.

${ }^{8}$ Professora Assistente de Reumatologia do HUEC Curitiba (PR) - Brasil

Endereço para correspondência: Luis Augusto Arana Rua Des. Otávio do Amaral, 770 Apto. 17 - Curitiba (PR) CEP 80730-400

E-mail: luisarana79@hotmail.com

Recebido para publicação em 03.11.2009

Aprovação em 11.12.2009

Nota Editorial: Depois de concluída a análise do artigo sob sigilo editorial e com a anuência do Dr. Luiz Carlos Molinari Gomes sobre a divulgação de seu nome como revisor, agradecemos sua participação neste processo.

\section{RESUMO}

Objetivo: Analisar a camada de fibras nervosas nos usuários de cloroquina com a tomografia de coerência óptica (OCT). Métodos: A espessura da camada de fibras nervosas foi mensurada pelo Stratus OCT ${ }^{\circledR}$ através do protocolo scan rápido da camada de fibras nervosas em 94 olhos de 48 pacientes usuários de cloroquina a pelo menos um ano e sem alteração macular aparente. A dose cumulativa e dose diária máxima ( $\mathrm{mg} / \mathrm{kg} / \mathrm{dia}$ ) e o tempo de uso da cloroquina foram correlacionadas com a espessura da camada de fibras nervosas. Os dados formam comparados com um grupo controle de 30 pacientes não usuários de cloroquina. Todos os pacientes foram submetidos a um exame oftalmológico completo incluindo medida da acuidade visual, refração, pressão intraocular, fundoscopia, retinografia e medida da escavação de papila. Resultados: Comparação entre a média da espessura da camada de fibras nervosas entre usuários do antimalárico $(107,60 \pm 13,25 \mu \mathrm{m})$ e o grupo controle $(99,05 \pm 13,08 \mu \mathrm{m})$ teve uma associação estatisticamente significativa $(\mathrm{p}=0,0137)$. Também foi encontrada essa associação entre os quadrantes temporal, superior e inferior da camada de fibras nervosas peripapilar dos dois grupos. Ao analisar a camada de fibras nervosas através das $12 \mathrm{~h}$ do relógio detectou-se pelo menos um defeito focal em mais de $50 \%$ dos usuários da medicação. Nos pacientes com o defeito focal foi observado uma relação com a dose diária máxima ( $p=0,0120)$. Conclusão: A tomografia de coerência óptica demonstrouse eficaz na detecção da diminuição da camada de fibras nervosas em usuários de cloroquina sem alterações fundoscópicas aparentes. Dessa forma, a tomografia de coerência óptica pode contribuir para o diagnóstico mais precoce da retinopatia por cloroquina.

Descritores: Tomografia de coerência óptica; Fibras nervosas; Retina; Cloroquina/efeitos adversos; Hidroxicloroquina/efeitos adversos; Células ganglionares da retina; Doenças retinianas/prevenção \& controle

\section{INTRODUÇÃ̃O}

Os antimaláricos (cloroquina e hidroxicloroquina) estãos incorporados ao tratamento de diversas desordens imunológicas. Entretanto, seu uso crônico pode acarretar uma degeneração retiniana irreversível que pode evoluir mesmo com a suspensão da medicação ${ }^{(1-2)}$. A cloroquina se une à melanina do epitélio pigmentar retiniano e essa junção talvez sirva para concentrar e prolongar a ação tóxica desse agente. Entretanto, a exata 
fisiopatogenia da retinopatia ainda não foi elucidada. Estudos iniciais realizados por alguns autores ${ }^{(3)}$ indicam que a cloroquina injetada parenteralmente em macacos Rhesus pode causar alterações significativas na camada de células ganglionares mostradas por histopatologia, mesmo na ausência de achados clínicos retinianos, testes eletrofisiológicos e angiografia fluoresceínica (AF). A droga tem a habilidade de alterar o $\mathrm{pH}$ dos compartimentos intracelulares e a função do epitélio pigmentar retiniano (EPR), o que gera a atrofia do epitélio( ${ }^{(4)}$.

As primeiras descrições da retinopatia por antimaláricos foram feitas por Hobbs ${ }^{(5)} \mathrm{em} 1959$. A toxicidade é caracterizada por alteração pigmentar bilateral na mácula geralmente poupando a fóvea, conhecida como maculopatia em "bull's eye". Segundo a Academia Americana de Oftalmologia os principais fatores para a toxicidade retiniana são a dose diária máxima da cloroquina (mais que $3 \mathrm{mg} / \mathrm{kg} / \mathrm{dia}$ ) e o tempo de uso, mas também se deve levar em consideração idade, doença renal e hepática associada ${ }^{(6)}$. Atualmente existem diversos exames de detecção da maculopatia como retinografia, angiografia, teste de visão de cores, campo visual, eletrorretinograma, eletrorretinograma multifocal, eletro-oculograma e autofluorescência ${ }^{(6-7)}$. Entretanto, ainda não existe um teste considerado padrãoouro para a sua detetecção precoce ${ }^{(6,8)}$.

A única conduta para a maculopatia por antimaláricos é a suspensão do medicamento; logo é necessário um exame de fácil manuseio e entendimento para detectar precocemente essa alteração. Como achados em modelo animal sugerem que a cloroquina afeta inicialmente as células ganglionares ${ }^{(3)}$, hipotetizamos que a estrutura da camada de fibras nervosas (CFN) (células ganglionares e seus axônios) possa ser precocemente comprometida pelo antimalárico. Assim, utilizamos a tomografia de coerência óptica (OCT) para mensurar a espessura da CFN peripapilar em usários de antimaláricos e num grupo controle.

\section{MÉTODOS}

Este estudo incluiu pacientes usuários de difosfato de cloroquina e sem alteração fundoscópica, nos quais foi levantado a dose cumulativa e diária $\mathrm{mg} / \mathrm{kg} / \mathrm{dia}$ da medicação (difosfato de cloroquina). Todos foram recrutados prospectivamente do ambulatório de Reumatologia do Hospital Universitário Evangélico de Curitiba (HUEC) entre maio de 2008 e outubro de 2008. Para o grupo controle formam incluídos indivíduos sem o uso de cloroquina, com idade e sexo semelhantes. Este trabalho foi aprovado pelo Comitê de Ética em Pesquisa do HUEC sendo obtido consentimento livre e esclarecido de todos os participantes.

Foram estudados 94 olhos de 48 usuários de cloroquina sem maculopatia e 30 pacientes (60 olhos) saudáveis para o grupo controle. Todos foram submetidos a um exame oftalmoscópico completo, incluindo medida da acuidade visual (AV), refração, pressão intraocular (PIO), fundoscopia e medida da escavação de papila. Os critérios de inclusão foram: idade acima de 18 anos, PIO menor que $22 \mathrm{mmHg}$, acuidade visual corrigida melhor que 20/40, uso de antimalárico por mais de 12 meses.

Indivíduos com evidência clínica de doença macular, cirurgia retiniana, doença neurológica ou diabetes foram excluídos. Além disso, os critérios de exclusão foram erro refracional maior que $5 \mathrm{DE}$ ou $2 \mathrm{DC}$, opacidades no eixo visual e sinais de glaucoma ("notch", Hoyt, aumento da escavação de papila maior que 0,6 ou assimetria entre as escavações acima de 0,2$)^{(9)}$.

O OCT foi utilizado para medir a camada de fibras nervosas, neste trabalho foi utilizado o Stratus OCT $^{\circledR}$ (Carl Zeiss Meditec, Inc.). O protocolo da rápida CFN 3.4 scan foi utilizado para quantificar a CFN peripapilar (software versão 4.0.1). Um círculo de 3,46 mm de diâmetro constituído de 256 A-scans foi posicionado manualmente no centro do disco óptico. A média, os quadrantes e as horas do relógio da espessura da CFN foram constatatdos na análise após o resultado das médias de três sequências circulares do scan. Todas as imagens escolhidas apresentaram graduação de no mínimo 7 . Em relação à classificação normativa foi divida em 4 categorias: 95 a $100 \%$ foi considerado hipernormal (cor branca no mapa); 5 a 95\% foi normal (verde); 1 a 5\% foi boderline (amarelo); e menos que $1 \%$ foi considerado anormal (vermelho). Para este estudo branco e verde foram englobadas como normais enquanto amarelo e vermelho foram classificadas como anormais. A análise do OCT pelas horas do relógio foi baseada no olho direito, assim $9 \mathrm{~h}$ do scan peripapilar representou o lado temporal do disco óptico de ambos os olhos.

Para a análise estatística foram utilizados os testes de Fisher e Qui-quadrado para os dados nominais; já os dados numéricos foram submetidos aos testes de Mann Whitney. Para fins estatísticos foram considerados os valores médios entre o olho direito (OD) e esquerdo (OE). A significância adotada foi de $5 \%$.

\section{RESULTADOS}

As características clínicas dos usuários de cloroquina e a média da CFN no OD e OE são demonstradas na tabela 1.

Dos 48 pacientes estudados, 45 eram do sexo feminino e 3 do masculino; já o grupo controle constituiu-se de $28 \mathrm{mu}-$ lheres e 2 homens. A média de idade dos usuários de cloroquina e do grupo controle foi 44,77 $\pm 12,9$ e 45,76 $\pm 12,61$ anos, respectivamente, não havendo diferença significativa entre os grupos $(\mathrm{p}=0,90)$. Dos 48 pacientes usuários de cloroquina 32 eram portadores de lúpus eritematoso sistêmico (LES), 7 de artrite reumatóide (AR), 7 de artrose e 2 de artrite reumatóide juvenil (ARJ). A exposição média à droga foi de 51,73 $\pm 26,9$ meses com dose cumulativa e total diária de $226,6 \pm 89,1 \mathrm{~g}$ e $1,710 \pm 0,509 \mathrm{mg} / \mathrm{kg} / \mathrm{dia}$, respectivamente.

Em relação à média da $\mathrm{CFN}$ foi constatado 107,60 $\pm 13,25 \mu \mathrm{m}$ no grupo controle e 99,05 $\pm 13,08 \mu \mathrm{m}$ nos usuários do medicamento ( $\mathrm{p}=0,0137)$. Além disso, foi encontrada uma diminuição da CFN estatisticamente significativa entre o grupo controle e 


\begin{tabular}{|c|c|c|c|c|c|c|c|c|c|}
\hline Número & Idade & Sexo & $\begin{array}{l}\text { Doença } \\
\text { de base }\end{array}$ & $\begin{array}{c}\text { Tempo em } \\
\text { meses }\end{array}$ & Dose & $\begin{array}{l}\text { cumulativa } \\
\text { (g) }\end{array}$ & $\begin{array}{c}\text { Dose máxima diária } \\
\text { em } \mathrm{mg} / \mathrm{kg} / \mathrm{dia}\end{array}$ & CFN OD & CFN OE \\
\hline 1 & 32 & $\mathrm{~F}$ & LES & 12 & & 36,00 & 1,589 & 140 & 132 \\
\hline 2 & 44 & $\mathrm{~F}$ & LES & 12 & & 45,00 & 1,875 & 120 & 116 \\
\hline 3 & 25 & $\mathrm{~F}$ & LES & 13 & & 51,83 & 1,629 & 93 & 90 \\
\hline 4 & 18 & $\mathrm{~F}$ & ARJ & 32 & & 63,15 & 2,020 & 121 & 115 \\
\hline 5 & 54 & M & LES & 26 & & 63,90 & 2,215 & 87 & 79 \\
\hline 6 & 61 & $\mathrm{~F}$ & Artrose & 33 & & 79,03 & 1,282 & 90 & 96 \\
\hline 7 & 63 & $\mathrm{~F}$ & Artrose & 22 & & 99,00 & 1,775 & 97 & 98 \\
\hline 8 & 68 & M & AR & 28 & & 108,00 & 2,205 & 89 & 94 \\
\hline 9 & 32 & $\mathrm{~F}$ & $\mathrm{AR}$ & 24 & & 109,50 & 2,000 & 100 & 99 \\
\hline 10 & 51 & $\mathrm{~F}$ & LES & 24 & & 109,50 & 2,238 & 101 & 106 \\
\hline 11 & 51 & $\mathrm{~F}$ & AR & 24 & & 109,75 & 2,283 & 100 & 100 \\
\hline 12 & 27 & $\mathrm{~F}$ & LES & 26 & & 110,38 & 1,024 & 105 & 104 \\
\hline 13 & 56 & $\mathrm{~F}$ & Artrose & 26 & & 114,65 & 1,304 & 91 & 85 \\
\hline 14 & 45 & $F$ & Artrose & 29 & & 114,93 & 0,857 & 92 & 89 \\
\hline 15 & 40 & $\mathrm{~F}$ & AR & 36 & & 164,25 & 2,222 & 113 & 108 \\
\hline 16 & 67 & $F$ & Artrose & 36 & & 164,25 & 1,724 & 89 & 83 \\
\hline 17 & 38 & $\mathrm{~F}$ & LES & 49 & & 178,23 & 1,247 & 116 & 99 \\
\hline 18 & 31 & $\mathrm{~F}$ & LES & 46 & & 186,75 & 0,877 & 107 & 102 \\
\hline 19 & 61 & $\mathrm{~F}$ & Artrose & 43 & & 193,50 & 2,459 & 95 & 87 \\
\hline 20 & 29 & $\mathrm{~F}$ & LES & 45 & & 202,50 & 2,840 & 105 & 107 \\
\hline 21 & 43 & $\mathrm{~F}$ & LES & 44 & & 203,25 & 1,027 & 91 & 97 \\
\hline 22 & 33 & $\mathrm{~F}$ & LES & 46 & & 207,00 & 2,013 & 114 & 107 \\
\hline 23 & 74 & $\mathrm{~F}$ & AR & 47 & & 211,50 & 2,057 & 100 & 112 \\
\hline 24 & 31 & $\mathrm{~F}$ & ARJ & 51 & & 229,50 & 2,290 & 75 & 82 \\
\hline 25 & 39 & $\mathrm{~F}$ & LES & 70 & & 235,04 & 0,980 & 101 & 96 \\
\hline 26 & 42 & $\mathrm{~F}$ & LES & 55 & & 238,60 & 1,072 & 83 & 101 \\
\hline 27 & 54 & $\mathrm{~F}$ & LES & 64 & & 245,94 & 0,914 & 85 & 93 \\
\hline 28 & 40 & $\mathrm{~F}$ & LES & 37 & & 246,28 & 2,412 & 79 & 78 \\
\hline 29 & 32 & $\mathrm{~F}$ & LES & 36 & & 246,35 & 1,127 & 89 & 91 \\
\hline 30 & 30 & $\mathrm{~F}$ & LES & 63 & & 254,58 & 1,271 & 104 & 104 \\
\hline 31 & 37 & $\mathrm{~F}$ & LES & 75 & & 272,98 & 1,111 & 117 & 114 \\
\hline 32 & 59 & $\mathrm{~F}$ & LES & 67 & & 280,23 & 0,959 & 110 & 121 \\
\hline 33 & 51 & $\mathrm{~F}$ & LES & 63 & & 280,58 & 2,112 & 95 & 93 \\
\hline 34 & 41 & $\mathrm{~F}$ & LES & 68 & & 291,95 & 0,920 & 95 & 100 \\
\hline 35 & 49 & $\mathrm{~F}$ & LES & 91 & & 294,23 & 1,286 & 120 & 114 \\
\hline 36 & 33 & $\mathrm{~F}$ & LES & 68 & & 303,78 & 0,867 & 90 & 84 \\
\hline 37 & 60 & $\mathrm{~F}$ & LES & 83 & & 313,43 & 0,750 & 64 & 58 \\
\hline 38 & 48 & $\mathrm{~F}$ & LES & 84 & & 314,37 & 1,612 & 101 & $x$ \\
\hline 39 & 59 & $M$ & AR & 70 & & 315,00 & 2,027 & 99 & $X$ \\
\hline 40 & 49 & $\mathrm{~F}$ & LES & 71 & & 316,68 & 1,196 & 97 & 91 \\
\hline 41 & 37 & $\mathrm{~F}$ & LES & 80 & & 319,25 & 0,961 & 104 & 99 \\
\hline 42 & 47 & $\mathrm{~F}$ & AR & 72 & & 324,00 & 2,013 & 128 & 128 \\
\hline 43 & 56 & $\mathrm{~F}$ & Artrose & 72 & & 328,50 & 1,332 & 110 & 115 \\
\hline 44 & 46 & $\mathrm{~F}$ & LES & 76 & & 380,25 & 1,056 & 100 & 99 \\
\hline 45 & 54 & $\mathrm{~F}$ & LES & 93 & & 383,50 & 1,027 & 100 & 100 \\
\hline 46 & 29 & $\mathrm{~F}$ & LES & 85 & & 387,50 & 1,918 & 94 & 89 \\
\hline 47 & 43 & $\mathrm{~F}$ & LES & 102 & & 392,25 & 1,562 & 108 & 100 \\
\hline 48 & 27 & $\mathrm{~F}$ & LES & 127 & & 563,25 & 0,951 & 106 & 96 \\
\hline
\end{tabular}

os usuários de cloroquina na média nos quadrantes temporal, superior e inferior (Tabela 2).

No estudo das $12 \mathrm{~h}$ do relógio foi encontrado pelo menos um defeito focal em 4 indivíduos (13\%) do grupo controle e em
25 pacientes $(52,08 \%)$ expostos à droga. Desses, 32\% apresentaram 3 ou mais defeitos focais na camada de fibras nervosas pelo OCT. Dos 25 pacientes com defeitos focais, 56\% apresentaram pelo menos um defeito nas $3 \mathrm{~h}$ do relógio que 
constituem a porção temporal peripapilar $-8,9$ e $10 \mathrm{~h} . \mathrm{Na}$ figura 1 verifica-se a análise da CFN pelo Stratus OCT num paciente com defeito nas $3 \mathrm{~h}$ do relógio que constituem o quadrante temporal $(8,9$ e $10 \mathrm{~h})$ além de um segmento do quadrante inferior que corresponde às $7 \mathrm{~h}$. Além do mapa básico do dado normativo da CFN das $12 \mathrm{~h}$ do relógio e dos 4 quadrantes o OCT fornece o perfil da CFN e a fotografia da papila com uma linha branca identificando o local correspondente à varredura da CFN.

A única associação estatisticamente positiva detectada entre tempo de uso da medicação, dose cumulativa, dose diária máxima com a espessura da CFN foi na dose diária máxima no grupo de pacientes com defeitos focais na camada peripapilar $(1,767 \pm 0,547 \mathrm{mg} / \mathrm{kg} /$ dia no grupo com defeitos versus $1,354 \pm$ 0,442 no grupo sem defeito) $\operatorname{com} \mathrm{p}=0,0120$.

\section{DISCUSSÃO}

A tomografia de coerência óptica é uma técnica que propicia um corte histológico do tecido retiniano. O OCT Stratus (time domain, resolução axial $10 \mu \mathrm{m}$ ) instituído neste trabalho

\begin{tabular}{|c|c|c|c|}
\hline & $\begin{array}{l}\text { CFN nos usuários } \\
\text { de Cloroquina }\end{array}$ & $\begin{array}{c}\text { CFN no } \\
\text { grupo controle }\end{array}$ & Valor do $\mathrm{p}$ \\
\hline Média & $99,05 \pm 13,08$ & $107,60 \pm 13,25$ & 0,0137 \\
\hline Temporal & $66,65 \pm 14,99$ & $76,77 \pm 11,29$ & 0,0009 \\
\hline Superior & $123,00 \pm 20,54$ & $133,90 \pm 17,29$ & 0,0192 \\
\hline Nasal & $76,90 \pm 19,46$ & $80,95 \pm 18,16$ & 0,2832 \\
\hline Inferior & $129,50 \pm 19,41$ & $138,50 \pm 17,41$ & 0,0452 \\
\hline \multicolumn{4}{|c|}{$\mathrm{P}=$ Mann Whitney } \\
\hline
\end{tabular}

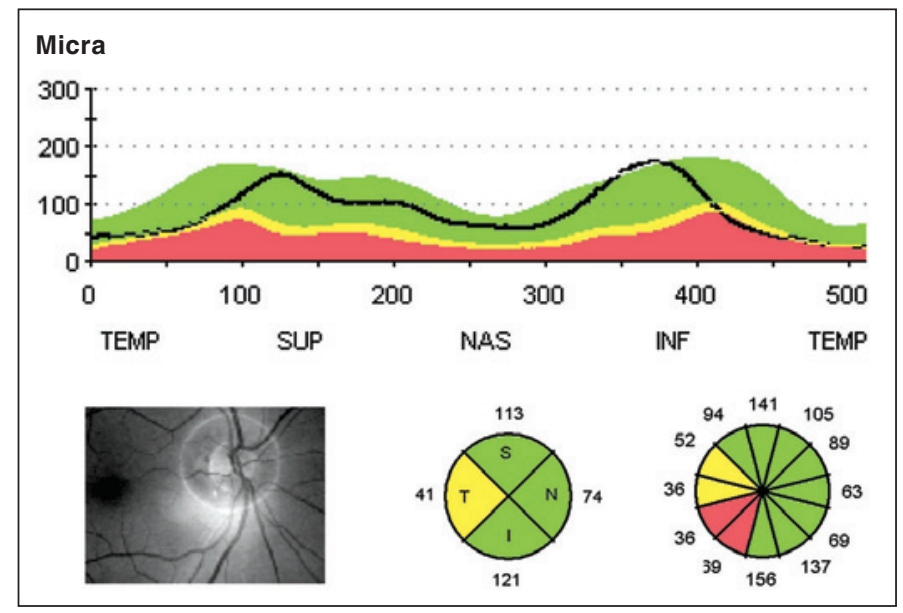

Figura 1 - Análise da CFN peripapilar pelo OCT; A) Perfil da CFN evidenciando sua diminuição no setor temporal; B) Imagem da papila com o scan indicando o local da tomografia; C) O mapa base dos dados normativos quantificando o defeito nas $4 \mathrm{~h}$ do relógio e no quadrante temporal. é amplamente utilizado na prática oftalmológica. O OCT apresenta inúmeras vantagens em relação aos atuais exames para o diagnóstico da retinopatia. Por exemplo, é um exame não invasivo (diferente da angiografia fluorescente); de fácil manuseio e entendimento (ao contrário do ERG multifocal); rápido e confortável ao paciente (diferente do campo visual) e, por fim, é um equipamento em constante evolução tecnológica e cada vez mais imbutido na oftalmologia.

A ocorrência de efeitos colaterais oculares potencialmente graves associado ao uso dos antimaláricos são minimizados pela conscientização dos reumatologistas e oftalmologistas. Entretanto, nosso estudo com a tomografia de coerência óptica demonstra alterações quantitativas entre a CFN peripapilar do grupo controle e usuários do antimalárico mesmo sem alterações fundoscópicas de maculopatia. Assim, os autores acreditam que o OCT possa encontrar alterações previamente ao dano do EPR detectado no mapeamento de retina. Esses achados são consistentes com estudos prévios da análise da CFN tanto pelo GDX quanto pelo OCT ${ }^{(10-13)}$.

Em nosso estudo mais de $50 \%$ dos usuários do medicamento apresentaram em pelo menos uma das $12 \mathrm{~h}$ do relógio coloração amarela ou vermelha pelo mapa normativo do OCT, o que os qualifica com defeito focal da CFN. A principal topografia afetada foi o quadrante temporal $(\mathrm{p}=0,0009)$. Isso pode ser explicado pela predominância das fibras que constituem a CFN peripapilar temporal serem provenientes do polo posterior, região na qual ocorrem as alterações fundoscópicas da retinopatia. Os autores acreditam que a análise da CFN, especialmente a temporal possa ser um dado relevante no acompanhamento dos usuários crônicos de cloroquina mesmo com ausência de alterações fundoscópicas.

Alguns estudos relatam que através do GDX ${ }^{\circledR}$ - polarimetria a laser - existe uma associação entre altas doses diárias de cloroquina com diminuição da CFN; entretanto, essa relação não foi verdadeira ao se comparar com a dose cumulativa ${ }^{(13)}$. Já no nosso estudo quando comparado a espessura da CFN com o tempo de uso da medicação, dose cumulativa e dose diária máxima foi encontrado uma correlação positiva apenas nos pacientes com defeitos focais na CFN e dose diária.

A dose diária máxima preconizada para evitar a maculopatia é abaixo de $3 \mathrm{mg} / \mathrm{kg} / \mathrm{dia}^{(6)}$. Nenhum dos 48 pacientes inclú́dos neste estudo apresentou a dose acima do preconizado, pois após 2 anos é norma do Ambulatório de Reumatologia do HUEC utilizar a droga em dias alternados. Isso também contribui para diminuir tanto a dose diária máxima quanto cumulativa desses pacientes. Não foi encontrada relação entre a CFN média com a dose diária máxima, cumulativa e tempo de exposição à droga; entretanto, ao analisar os pacientes com defeito focal foi verificado uma relação significamente estatística com a dose máxima diária $(\mathrm{p}=0,0120)$. Embora se acredite que a dose cumulativa, tempo de uso e dose diária são importantes para desencadear a retinopatia ${ }^{(1)}$, estudos recentes ressaltam a importância da dose diária ${ }^{(6,14)}$ pois a posologia de $150 \mathrm{mg}$ ao dia de cloroquina tem um efeito tóxico diferente ao se comparar uma pessoa de $45 \mathrm{~kg}$ versus $100 \mathrm{~kg}$. 


\section{CONCLUSÃO}

O OCT é capaz de detectar a diminuição da CFN em pacientes usuários de cloroquina sem alterações fundoscópicas aparentes. Estudos futuros, entretanto, são imperativos para verificar o valor preditivo dessa tecnologia para o diagnóstico precoce da maculopatia.

\section{ABSTRACT}

Purpose: To evaluated the retinal nerve fiber layer in chloroquine patients with optical coherence tomography (OCT). Methods: Nerve fibers layer thickness was measured by a proprietary OCT Stratus ${ }^{\circledR}$ through the fast retinal nerve fiber layer protocol in ninety-four eyes of 48 patients exposed to chloroquine for at least one year with no apparent macular disorder. Cumulative dose, maximum daily dose $(\mathrm{mg} / \mathrm{kg} /$ day) and time of chloroquine use were correlated with the retinal nerve fiber layer thickness. Data were compared with a control group of 30 patients who did not use chloroquine. All patients underwent a complete ophthalmologic examination including visual acuity, refraction, intraocular pressure, fundoscopy, angiography and measurement of cup-to-disc ratio. Results: The average thickness of retinal nerve fiber layer between antimalaric users $(107.60 \pm 13.25 \mu \mathrm{m})$ and the control group $(99.05 \pm 13.08 \mu \mathrm{m})$ had a statistically significant difference $(\mathrm{p}=0.0137)$. Furthermore, a positive association of the peripapillary thickness measurements between temporal, upper and lower quadrants of both groups was found. Twelve clock hours retinal nerve fiber layer analysis detected at least one focal defect in more than $50 \%$ of the medication users. Particularly, a statistical correlation with the maximum daily dose was found in patients with focal defects $(\mathrm{p}=0.0120)$. Conclusion: Optical coherence tomography is effective in detecting nerve fiber layer loss in chloroquine patients without apparent fundus changes. Thus, optical coherence tomography may contribute to the early diagnosis of chloroquine retinopathy.
Keywords: Tomography, optical coherence; Nerve fibers; Retina; Chloroquine/adverse effects; Hydroxychloroquine/adverse effects; Retinal ganglion cells; Retinal diseases/prevention \& control

\section{REFERÊNCIAS}

1. Browning DJ. Hydroxychloroquine and chloroquine retinopathy: screening for drug toxicity. Am J Ophthalmol. 2002;133(5):649-56. Comment in: Am J Ophthalmol. 2002;134(6):935; author reply 935-6.

2. Shinjo SK, Maia OO Jr, Tizziani VA, Morita C, Kochen JA, Takahashi WY, et al. Chloroquine-induced bull's eye maculopathy in rheumatoid arthritis: related to disease duration? Clin Rheumatol. 2007;26(8):1248-53.

3. Rosenthal AR, Kolb H, Bergsma D, Huxsoll D, Hopkins JL. Chloroquine retinopathy in the rhesus monkey. Invest Ophthalmol Vis Sci. 1978;17(12): 1158-75.

4. Mahon GJ, Anderson HR, Gardiner TA, McFarlane S, Archer DB, Stitt AW. Chloroquine causes lysosomal dysfunction in neural retina and RPE: implications for retinopathy. Curr Eye Res. 2004;28(4):277-84.

5. Hobbs HE, Sorsby A, Friedman A. Retinopathy following chloroquine therapy. Lancet.1959;2(7101):478-80.

6. Marmor MF, Carr RE, Easterbrook M, Farjo AA, Mieler WF; American Academy of Ophthalmology. Recommendations on screening for chloroquine and hydroxychloroquine retinopathy: a report by the American Academy of Ophthalmology. Ophthalmology. 2002;109(7):1377-82.

7. Kellner U, Kellner S, Weinitz S. Chloroquine retinopathy: lipofuscin- and melanin-related fundus autofluorescence, optical coherence tomography and multifocal electroretinography. Doc Ophthalmol.2008;116(2):119-27.

8. Kellner U, Renner AB, Tillack H. Fundus autofluorescence and mfERG for early detection of retinal alterations in patients using chloroquine/hydroxychloroquine. Invest Ophthalmol Vis Sci. 2006;47(8):3531-8.

9. Sung KR, Kim DY, Park SB, Kook MS. Comparison of retinal nerve fiber layer thickness measured by Cirrus HD and Stratus optical coherence tomography. Ophthalmology. 2009;116(7):1264-70.

10. Rodriguez-Padilha JA, Hedges TR $3^{\text {rd }}$, Monson B, Srinivasan V, Wojtkowski M, Reichel E, et al. High-speed ultra-high-resolution optical coherence tomography findings in hydroxychloroquine retinopathy. Arch Ophthalmol. 2007; 125(6):775-80.

11. Pasadhika S, Fishman GA. Effects of chronic exposure to hydroxychloroquine or chloroquine on inner retinal structures. Eye (Lond). 2009; Apr 17 (Epub ahead of print).

12. Antunes DAL, Cavalcanti HD, Leal D, Santana HV, Santos RAV, Santos LPF. Alteração da camada de fibras nervosas da retina em usuários crônicos de cloroquina. Arq Bras Oftalmol. 2005;68(2):195-7.

13. Bonanomi MT, Dantas NC, Medeiros FA. Retinal nerve fibre layer thickness measurements in patients using chloroquine. Clin Experiment Ophthalmol. 2006;34(2):130-6.

14. Mackenzie AH. Dose refinements in long-term therapy of rheumatoid arthritis with antimalarials. Am J Med. 1983;75(1A):40-5.

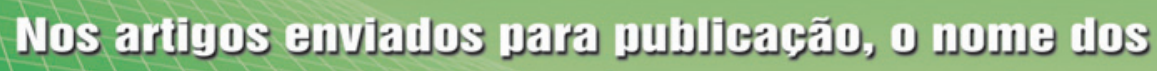

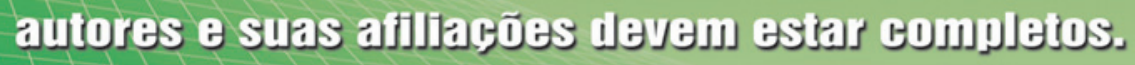

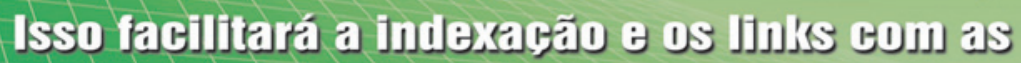

bases do dajus o o CIJ Lajijos. 